\title{
AVALIAÇÃO DO DIAGNÓSTICO DE ENFERMAGEM “DOR AGUDA” EM PACIENTES INTERNADOS EM UMA CLÍNICA MÉDICO-CIRÚRGICA
}

\section{EVALUATION OF THE NURSING DIAGNOSIS "ACUTE PAIN" IN HOSPITALIZED PACIENTS IN A MEDICAL- SURGICAL CLINIC}

\section{EVALUACIÓN DEL DIAGNÓSTICO DE ENFERMERÍA "DOLOR AGUDO" EN PACIENTES INTERNADOS EN UNA CLÍNICA MÉDICA-QUIRÚRGICA}

Gabriela Tavares Boscarol ${ }^{1}$, Camila Santana Domingos ${ }^{2}$, Cristiane Chaves de Souza ${ }^{3}$, Meire Chucre Tannure ${ }^{4}$, Tânia Couto Machado Chianca ${ }^{5}$, Patrícia Oliveira Salgado ${ }^{6}$.

\begin{abstract}
RESUMO
Objetivo: avaliar o diagnóstico de enfermagem “Dor aguda" em pacientes internados, em uma unidade de clínica médico-cirúrgica, a partir do uso de um software. Método: estudo quantitativo do tipo transversal, observacional e descritivo com pacientes internados em uma unidade de clínica médico-cirúrgica masculina e feminina de um hospital de ensino. A amostra foi composta por 100 pacientes. Os dados foram coletados por meio de entrevista previamente estruturada em um software. Na análise descritiva dos dados, calculou-se a taxa de prevalência e correlação de Pearson. Considerou-se nível de significância de $5 \%$. Resultados: prevalência de 34\% do diagnóstico de enfermagem "Dor aguda". A maioria dos pacientes tinha menos que 60 anos e era do sexo masculino. Nas últimas avaliações, 21 (61,8\%) pacientes mantiveram queixa de dor, sobretudo, relacionada a procedimentos cirúrgicos e traumas. Verificou-se que a proporção de homens foi maior que a de mulheres na queixa de dor leve e moderada. Quanto maior o tempo de internação maior foi o número de pacientes com a queixa de dor resolvida ( $r=0,384 ; p=$ 0,025). Conclusão: os dados deste estudo corroboram com os achados de outros autores no que tange à importância da avaliação da dor e intervenções adequadas em seu manejo.

Descritores: Enfermagem; Processo de Enfermagem; Software; Diagnóstico de Enfermagem; Dor aguda.
\end{abstract}

\section{ABSTRACT}

Objective: to evaluate the nursing diagnosis "Acute pain" in patients hospitalized in a medical-surgical clinic unit using a software. Methods: quantitative, cross-sectional, observational and descriptive study with patients hospitalized in a male and female medical-surgical clinic of a teaching hospital. The sample consisted of 100 patients. The data were collected based on a previously structured interview using a software. The Pearson's correlation test and the prevalence rate were calculated in the descriptive data analysis. A significance level of $5 \%$ was considered. Results: $34 \%$ prevalence of the nursing diagnosis "Acute pain". Most patients were less than 60 years old and were male. In the last evaluations, 21 (61.8\%) patients maintained pain complaints, mainly related to surgical procedures and trauma. The proportion of men was greater than that of women in the complaint of mild and moderate pain. The longer the hospitalization time, the greater the number of patients with resolved pain complaint $(r=0.384, p=$ 0.025). Conclusions: the data found in this study corroborate the findings of other authors regarding the importance of pain assessment and appropriate interventions in its management.

Descriptors: Nursing; Nursing Process; Software; Nursing Diagnosis; Acute Pain.

\section{RESUMEN}

Objetivo: evaluar el diagnóstico de enfermería "Dolor agudo" en pacientes internados en una unidad clínica médico-quirúrgica a partir del uso de un software. Métodos: estudio cuantitativo del tipo transversal, observacional y descriptivo con pacientes internados en una unidad clínica médico-quirúrgica masculina y femenina, de un hospital universitario . La muestra fue compuesta por 100 pacientes. Los datos fueron recolectados a través de una entrevista previamente estructurada con base en un software. En el análisis descriptivo de los datos, se calculó la tasa de prevalencia y la correlación de Pearson. Se consideró un nivel de significancia del 5\%. Resultados: prevalencia del 34\% del diagnóstico de enfermería "Dolor agudo". La mayoría de los pacientes tenían menos de 60 años y eran del sexo masculino. En la última evaluación 21 (61,8\%) pacientes mantuvieron queja de dolor, sobre todo relacionada a procedimientos quirúrgicos y traumas. Se verificó que la proporción de hombres fue mayor que la de las mujeres en cuanto a la queja de dolor leve y moderado. El mayor tiempo de internamiento resultó en mayor número de pacientes con la queja de dolor resuelta $(r=0,384, p=0,025)$. Conclusiones: los datos de este estudio corroboran los hallazgos de otros autores en lo que se refiere a la importancia de la evaluación del dolor e intervenciones adecuadas en su manejo.

Descriptores: Enfermería; Proceso de Enfermería; Programas informáticos; Diagnóstico de Enfermería; Dolor Agudo.

${ }^{1}$ Graduanda em Enfermagem pela Universidade Federal de Viçosa. ${ }^{2}$ Enfermeira, Mestre em Ciências da Saúde pela Universidade Federal de Viçosa. ${ }^{3}$ Enfermeira, Doutora em Enfermagem pela Universidade Federal de Minas Gerais. ${ }^{4}$ Enfermeira, Doutora em Enfermagem pela Universidade Federal de Minas Gerais. ${ }^{5}$ Enfermeira, Pós Doutora em Enfermagem pela Universidade de lowa. ${ }^{6}$ Enfermeira, Doutora em Enfermagem pela Universidade Federal de Minas Gerais.

Como citar este artigo:

Boscarol GT, Domingos CS, Souza CC, et al. Avaliação do diagnóstico de enfermagem "dor aguda" em pacientes internados em uma clínica médico-cirúrgica. Revista de Enfermagem do Centro oeste Mineiro. 2019;9:e3312. [Access___]; Available in:__. DOI: http://dx.doi.org/10.19175/recom.v9i0.3312 


\section{INTRODUÇÃO}

A Associação Internacional, para o Estudo da Dor (IASP, International Association for Study of Pain, 1994), define-a como "uma experiência sensitiva e emocional desagradável, associada a uma lesão tecidual, potencial ou descrita em termos de tal lesão"(1).

Segundo Souza (2016), a dor é considerada "multidimensional e inerente à existência humana, fazendo parte do processo vital de cada indivíduo". Refere-se, continuamente, aos ambientes de atenção à saúde e, com maior frequência, a pacientes hospitalizados ${ }^{(2)}$.

As dores podem ser classificadas de natureza aguda ou crônica de acordo com seu aspecto temporal. As dores agudas são consideradas um importante sinal de alerta e possuem duração limitada no tempo e espaço, cessando com o encontro da causa raiz. Já as dores crônicas se constituem em uma doença, um processo que persiste, mesmo após a restauração da condição sadia do indivíduo ${ }^{(3)}$.

À luz de uma visão mais humanística, a dor pode ser pensada como uma experiência sensorial e emocional que agrega dano tecidual real ou potencial a prejuízos culturais, psíquicos e físicos. Esse estado pode ser considerado como um dos principais causadores do sofrimento humano, comprometendo a qualidade de vida e trazendo consequências emocionais, cognitivas, sociais e culturais ao indivíduo acometido ${ }^{(3)}$.

O controle da dor deve ser uma inquietação do enfermeiro e, visando orientar o cuidado, durante assistência ao paciente, utilizase como instrumento metodológico o Processo de Enfermagem $(\mathrm{PE})^{(4)}$. Diante de um paciente com dor, o enfermeiro deve ser capaz de realizar a primeira etapa do $\mathrm{PE}$, por uma coleta de dados abrangente, com a descrição da experiência dolorosa e suas repercussões, no funcionamento biológico, emocional, espiritual, bem como elencar os fatores que contribuem para a sua melhora ou piora.

Os Diagnósticos de Enfermagem (DE) representam a segunda etapa do PE. A taxonomia NANDA-International (NANDA-I) definiu inicialmente o DE "Dor" como "uma experiência e relato de severo desconforto ou uma sensação desconfortável, não diferenciando a condição crônica da aguda" ${ }^{\prime(5)}$.

Em 1986, foi incluído "Dor crônica" como um DE para queixas de dor com duração superior a seis meses. Apenas 10 anos depois, o diagnóstico "Dor aguda" foi integrado à taxonomia e definido como "Experiência sensorial e emocional desagradável associada à lesão tissular real ou potencial, ou descrita em termos de tal lesão (International Association for the Study of Pain); início súbito ou lento de intensidade leve à intensa, com término antecipado ou previsível”(5).

Apesar DE "Dor aguda" existir há mais de 20 anos, estudo aponta que, independentemente do local onde se presta a assistência de enfermagem, há uma não adesão dos enfermeiros na elaboração de $\mathrm{DE}$, a incluir o de dor, problema comum no exercício clínico ${ }^{(6)}$. Essa realidade pode ser justificada pelo fato de que, na prática, os profissionais encontram dificuldade, ao implantar todas as etapas do PE, dada a sobrecarga de trabalho à assistência ou deficiência de conhecimento sobre o seu uso.

Destarte, o uso de softwares, para registro do $P E$, vem sendo indicado como uma ferramenta capaz de contribuir para a sua implantação, de forma mais rápida, precisa e completa, favorecendo maior disponibilidade dos enfermeiros, para as atividades assistenciais, maior contato com os pacientes $\mathrm{e}$ operacionalização das etapas do $\mathrm{PE}^{(6)}$. Pelo uso de softwares busca-se concretizar a implantação do $\mathrm{PE}$, integrando suas etapas, em uma estrutura lógica de dados, informação e conhecimento, para a tomada de decisão do cuidado de enfermagem, restabelecendo uma condição de saúde favorável ao paciente ${ }^{(6)}$.

Mediante os benefícios do uso das tecnologias de informação, para a implementação do $\mathrm{PE}$, está sendo desenvolvido um projeto maior intitulado "Avaliação da aplicabilidade de um software com o processo de enfermagem em unidades de internação". Nesse projeto, o software Sistema de Informação com o Processo de Enfermagem em Terapia Intensiva (SIPETI), que contém as etapas do PE fundamentado, na teoria das necessidades humanas básicas (NHB) de Wanda de Aguiar Horta, inicialmente desenvolvido para ser usado em uma unidade de terapia intensiva de adultos ${ }^{(6)}$, foi utilizado em uma unidade de clínica médico-cirúrgica para adaptação.

Durante a vivência de coleta de dados, utilizando o software, observou-se que a dor foi um problema comum identificado, em pacientes avaliados desde a admissão até a alta, transferência ou óbito, inquietando as pesquisadoras sobre os fatores a ela relacionados. 
Assim, delineou-se este estudo com o objetivo de avaliar O DE "dor aguda", em pacientes internados em uma unidade de clínica médico-cirúrgica, a partir do uso de um software.

\section{MÉTODOS}

Trata-se de um estudo quantitativo do tipo transversal, observacional e descritivo, realizado nas clínicas médico-cirúrgicas masculina e feminina de um hospital da Zona da Mata Mineira. As duas clínicas, onde o software foi empregado, somam 35 leitos destinados ao atendimento, em sua maioria, de pacientes do Sistema Único de Saúde (SUS) das especialidades clínicas médica, cirurgia geral e ortopedia. A instituição é reconhecida como hospital de ensino e classificado como de média complexidade.

A população do estudo foi composta por pacientes internados, nas clínicas médicocirúrgicas masculina e feminina, no período de 9 de janeiro a 17 de março de 2017, totalizando 271 pacientes. O tamanho da amostra foi determinado pelo método não probabilístico de amostragem de conveniência, que se caracteriza pelo uso de pessoas ou objetos mais prontamente acessíveis como sujeitos de pesquisa num estudo ${ }^{(7)}$. Foram incluídos ao estudo pacientes conscientes, de ambos os sexos, idade superior a 18 anos, internados nas clínicas e que aceitaram participar da pesquisa no momento em que as pesquisadoras realizavam a coleta de dados. Foram excluídos do estudo os pacientes que, no momento da coleta de dados, estavam sendo submetidos a exames, procedimentos invasivos, que saíram da unidade por motivo de alta, óbito ou transferência ou se recusaram a participar do estudo. Para aqueles pacientes que apresentavam algum déficit cognitivo, por idade ou condição de saúde, foi solicitado ao acompanhante a assinatura do TCLE. A amostra final foi composta por 100 pacientes que atenderam aos critérios de inclusão. Não houve perdas amostrais, pois todos os pacientes cadastrados foram acompanhados diariamente até a alta, óbito ou transferência.

Para a utilização do software, realizou-se um treinamento interobservadores, a fim de calibrar as pesquisadoras, para descrever o mesmo fenômeno observado, obtendo maior semelhança possível ${ }^{(8)}$. Após três ciclos de treinamentos, atingiu- se o índice de concordância acima de $90 \%$, recomendado por Coluci, Alexandre e Milani ${ }^{(8)}$, em que os registros foram considerados fidedignos e confiáveis, sendo as pesquisadoras consideradas treinadas e com habilidades necessárias para iniciar a coleta de dados.

Os dados foram coletados por entrevista, previamente estruturada, no software SIPETI, por duas pesquisadoras, à beira do leito do paciente, nos períodos matutino e vespertino. Na admissão do paciente, foi realizada a primeira avaliação com anamnese e exame físico completos. Nos dias subsequentes de internação, foram realizadas avaliações referentes ao exame físico somente, visto que as pesquisadoras tinham acesso ao histórico do paciente no software.

O questionamento sobre dor fazia parte do exame físico e era verificado diariamente com o paciente que respondia a perguntas sobre presença da dor, intensidade, local e tipo. Para a avaliação da intensidade da dor, foi utilizada a Escala Numérica de Dor (END), elaborada por Huskisson ${ }^{(9)}$, que varia de zero a 10 pontos, sendo zero ausência de dor e 10 a pior dor já sentida pelo paciente, considerando experiências dolorosas prévias. Para critérios de análise estatística descritiva, a intensidade da dor foi estratificada, utilizando a Escala Analgésica de Dor (EAD), elaborada pela $\mathrm{OMS}^{(10)}$, que categoriza em "ausência de dor", quando a intensidade relatada foi zero; "dor leve" de intensidade uma a três, "dor moderada" de intensidade quatro a sete e "dor severa" de intensidade maior que oito. O local e o tipo de dor foram avaliados por meio de relato verbal.

Os dados foram organizados no Excel ${ }^{\circ}$, versão 2010 e, após verificação de sua consistência, foram exportados para o Statistical Package for Social Sciences (SPSS), versão 23. A análise estatística descritiva dos resultados foi realizada por meio das frequências absolutas e relativas. Buscando investigar a relação entre o tempo de internação com o status do diagnóstico de dor, foi realizada a Correlação de Pearson. Para as demais variáveis, utilizou-se o teste quiquadrado de Pearson. Para critérios de decisão estatística, em todas as comparações, foi adotado o nível de significância $(\alpha)$ de $5 \%$.

Para a realização desta pesquisa, foi respeitada a Resolução do Conselho Nacional de Saúde (CNS) 466/12 que regulamenta as pesquisas envolvendo seres humanos no Brasil. $O$ projeto obteve aprovação do Comitê de Ética em Pesquisa da Universidade competente (Parecer Ético no 45113815.7.0000.5153). O anonimato dos pacientes foi garantido. Os objetivos e o caráter voluntário da pesquisa foram 
previamente esclarecidos a todos os participantes. Todos os pacientes ou representantes puderam participar do estudo após assinatura do TCLE.

\section{RESULTADOS E DISCUSSÃO}

Durante o período de dois meses consecutivos de utilização do SIPETI, nas clínicas médico-cirúrgicas masculina e feminina, foram avaliados 100 pacientes. A prevalência do DE "Dor aguda" foi de 34\%.

Estudos realizados em cenários semelhantes têm evidenciado altas frequências de $D E$, principalmente, quando se trata de pacientes em pós-operatório, variando entre $32,1 \%$ e $42,2 \%^{(11-12)}$. Uma pesquisa realizada, em uma unidade de pós-operatório imediato e mediato de cirurgia geral, mostrou que a dor aguda foi queixa de $42,2 \%$ entre os 99 pacientes avaliados ${ }^{(11)}$. Os autores reforçam a importância do manejo adequado da dor, bem como sua avaliação criteriosa, pois o sentimento de dor pode gerar um gasto de energia maior que o esperado e interferir negativamente na recuperação do paciente ${ }^{11}$. É importante que o enfermeiro tenha foco contínuo na queixa de dor do paciente para evitar ou minimizar a ocorrência de outros problemas de enfermagem ${ }^{(12)}$.

A idade dos pacientes variou entre 18 e 90 anos, sendo a maioria (31-91,2\%) com idade inferior a 60 anos e média de idade de 41,4 anos, sexo masculino (19-55,9\%), raça parda (20$58,9 \%)$ e com o 1 ㅇ grau Completo $(17-50,2 \%)$ (Tabela 1).

Tabela 1 - Caracterização dos participantes do estudo de acordo com a idade, sexo, raça autodeclarada e escolaridade, Viçosa-MG, 2017.

\begin{tabular}{|c|c|c|}
\hline Variáveis & $\mathbf{N}$ & $\%$ \\
\hline \multicolumn{3}{|l|}{ Idade(anos) } \\
\hline $18-30$ & 10 & 29,4 \\
\hline $31-60$ & 21 & 61,8 \\
\hline $61-90$ & 3 & 8,8 \\
\hline \multicolumn{3}{|l|}{ Sexo } \\
\hline Masculino & 19 & 55,9 \\
\hline Feminino & 15 & 44,1 \\
\hline \multicolumn{3}{|l|}{ Raça } \\
\hline Branca & 8 & 23,5 \\
\hline Parda & 20 & 58,9 \\
\hline Negra & 6 & 17,6 \\
\hline \multicolumn{3}{|l|}{ Escolaridade } \\
\hline Analfabeto & 2 & 5,8 \\
\hline 1ㅇ grau Incompleto & 5 & 14,7 \\
\hline 10 grau Completo & 17 & 50,2 \\
\hline $2 \circ$ grau incompleto & 3 & 8,8 \\
\hline 2ㅇ grau completo & 6 & 17,6 \\
\hline 3ㅇ grau incompleto & 0 & 0 \\
\hline 3ㅇ grau completo & 1 & 2,9 \\
\hline
\end{tabular}


O perfil dos participantes encontrados neste estudo divergiu de uma pesquisa que avaliou pacientes, em um cenário semelhante ${ }^{(13)}$ no que tange à faixa etária, sendo encontrada maior prevalência $(60 \%)$ em pacientes na faixa etária de 50 anos ou mais. Contudo, quanto ao sexo, a proporção de pacientes foi semelhante, $52 \%$ do sexo masculino e $48 \%$ do sexo feminino ${ }^{(13)}$.

Identificou-se que os sistemas orgânicos, causadores da internação, mais representados foram o gastrintestinal e musculoesquelético, em nove $(26,5 \%)$ pacientes, seguidos pelo cardiovascular e renal, em quatro (11,8\%) pacientes, reprodutor em três $(8,9 \%)$ pacientes, hematopoiético, hepático, metabólico, neurológico e respiratório em um (2,9\%) paciente cada.

Quanto à situação clínica, a maioria dos pacientes (21-61,8\%) estava internada por motivos clínicos. O tempo de internação variou entre um e 10 dias, sendo o tempo médio de 3,97 dias. Quanto ao desfecho clínico, 30 (88,2\%) pacientes tiveram alta hospitalar, dois $(5,9 \%)$ foram transferidos para outro setor hospitalar e dois $(5,9 \%)$ foram transferidos para outra unidade de internação.

Estudo que avaliou o perfil dos pacientes internados, em uma clínica médica ${ }^{(13)}$, encontrou grande prevalência de pacientes com doenças do aparelho cardiovascular (60\%), seguido de doenças respiratórias e neurológicas $(40 \%)$ e menor parcela com doenças infecciosas, como, por exemplo, sepse (12\%) e erisipela (8\%). Salienta-se que a literatura é escassa quanto a estudos que abordem os sistemas orgânicos que mais levam os pacientes à hospitalização, em cenários semelhantes ao desta pesquisa, o que dificulta a comparação com outros estudos. Entretanto verifica-se que esses dados são o reflexo do perfil de pacientes que são atendidos em cada serviço.

Apesar de terem sido realizadas intervenções, durante a internação, para minimizar ou eliminar a dor, 21 (61,8\%) pacientes mantiveram o quadro, no momento da última avaliação, realizada antes do desfecho e apenas $13(38,2 \%)$ tiveram o DE "Dor aguda" resolvido. Além disso, constatou-se que dos 34 pacientes com o DE "Dor aguda" apenas 17 (50\%) tiveram acesso a alguma forma de analgesia, sendo as classes medicamentosas mais utilizadas, para o alívio da dor, os analgésicos comuns $(15-88,2 \%)$, anti-inflamatórios não esteroides (AINE) (1270,5\%) e opioides (7-41,1\%).

Embora somente $50 \%$ dos pacientes tenham tido acesso a medidas farmacológicas de alívio da dor neste estudo, autores mostram que os medicamentos têm papel importante, no controle dessa queixa, quando prescritos com segurança. Revisão sistemática que identificou a eficácia dos medicamentos da classe AINE, no controle da dor associada à inflamação ou lesão tecidual, demonstrou que essa terapia evidenciou-se ainda melhor quando associada a opioides, reduzindo significativamente a prevalência da dor no período pós-operatório ${ }^{(14)}$.

Neste estudo observou-se que dos 17 pacientes que tiveram acesso à analgesia, a maioria (15-88,2\%) foi medicada com analgésicos comuns. De modo semelhante, estudo que avaliou a dor aguda, em pacientes da emergência, demonstrou que os analgésicos comuns representam a classe medicamentosa de primeira escolha e utilizada com maior frequência, tendo resolvido o quadro de dor de $25 \%$ dos pacientes ${ }^{(15)}$. Uma revisão sistemática, que avaliou a eficácia dos AINEs no controle da dor aguda, verificou que $68,7 \%$ dos artigos classificaram essa classe medicamentosa como "eficaz", no controle da dor aguda de intensidade leve à moderada, quando usada de forma isolada ou combinada com outros medicamentos, além de ser considerada segura por $56,2 \%$ dos autores ${ }^{(14)}$.

Além das medidas farmacológicas, vale ressaltar a importância da aplicação de medidas não farmacológicas para o alívio da dor. A aplicação de calor e frio, exercício, massagem, distração, relaxamento e conforto são alguns exemplos de medidas não farmacológicas. Uma revisão sistemática ${ }^{(16)}$ mostrou que as medidas farmacológicas e não farmacológicas se complementam no alívio da dor. O suporte emocional e a melhora do posicionamento no leito foram capazes de aliviar a dor em $88,5 \%$ e $75,8 \%$ dos pacientes, respectivamente ${ }^{(16)}$. A literatura apresenta que, apesar de fazer parte das competências do enfermeiro o manejo da dor, existe uma lacuna na formação quanto à gestão desse fenômeno, sendo necessário um maior investimento na capacitação dos profissionais dentro dessa temática ${ }^{(16)}$.

Ao avaliar os fatores relacionados, apresentados pela NANDA-I, para o diagnóstico de "Dor aguda", observou-se que mais da metade 
dos pacientes (19-55,9\%) foram submetidos à ação de agentes físicos causadores de lesão, ou seja, a queixa de dor esteve, em sua maioria, relacionada a procedimentos cirúrgicos e traumas. Verificou-se que a maioria dos pacientes
$(18-52,9 \%)$ queixou-se de dor moderada, na região abdominal $(10-29,4 \%)$, sendo relatada como contínua por seis $(17,7 \%)$ dos pacientes (Tabela 2).

Tabela 2 - Distribuição dos fatores relacionados, intensidade, localização e tipo da dor, Viçosa- MG, 2017.

\begin{tabular}{|c|c|c|}
\hline Variáveis & $N$ & $\%$ \\
\hline \multicolumn{3}{|l|}{ Fatores relacionados } \\
\hline Agentes lesivos físicos & 19 & 55,9 \\
\hline Agentes lesivos biológicos & 15 & 44,1 \\
\hline \multicolumn{3}{|l|}{ Intensidade da dor } \\
\hline Dor moderada & 18 & 52,9 \\
\hline Dor leve & 9 & 26,5 \\
\hline Dor severa & 7 & 20,6 \\
\hline \multicolumn{3}{|l|}{ Local da dor } \\
\hline Região abdominal & 10 & 29,4 \\
\hline Membros inferiores & 8 & 23,5 \\
\hline Membros superiores & 4 & 11,8 \\
\hline Região lombar & 4 & 11,8 \\
\hline Cabeça & 3 & 8,8 \\
\hline Região torácica & 3 & 8,8 \\
\hline Região geniturinária & 2 & 5,9 \\
\hline \multicolumn{3}{|l|}{ Tipo de dor } \\
\hline Não relatada & 7 & 20,6 \\
\hline Contínua & 6 & 17,7 \\
\hline Latejante & 5 & 14,8 \\
\hline Em queimação & 4 & 11,8 \\
\hline Em cólica & 3 & 8,8 \\
\hline Lancinante & 3 & 8,8 \\
\hline Súbita & 3 & 8,8 \\
\hline Cortante & 1 & 2,9 \\
\hline Em câimbra & 1 & 2,9 \\
\hline Formigamento & 1 & 2,9 \\
\hline
\end{tabular}

Fonte: Dados do estudo, 2017.

A maioria dos participantes deste estudo $(55,9 \%)$ relatou dor relacionada a agentes físicos, principalmente procedimentos cirúrgicos. Autores verificaram forte associação entre as alterações fisiológicas, causadas por cirurgias de grande porte, principalmente ortopédicas, e a presença de dor pós-operatória ${ }^{(17)}$. Embora a dor, no período pós-operatório, necessite de grande atenção e cuidados, seu manejo é inadequado, na maioria das vezes, tendo a equipe de enfermagem pouco envolvimento em sua avaliação e controle, sendo necessária maior capacitação dos profissionais para o uso de estratégias avançadas no cuidado com a dor ${ }^{(18)}$.

Percebeu-se que metade dos pacientes relata dor contínua, mesmo após a administração 
de medicamentos, para o seu controle. Esse dado reflete a necessidade de maior empoderamento da enfermagem em relação ao manejo da dor. Verificou-se, durante a condução deste estudo, que, em muitos casos, a equipe de enfermagem é dependente de uma prescrição médica, para intervir quanto à queixa de dor do paciente, esquecendo-se do seu papel na implementação de medidas não farmacológicas para o seu alívio.

É importante ressaltar que os pacientes que não necessitam de procedimento cirúrgico, também, podem experimentar a dor como resultado de sua condição de saúde e sofrer intercorrências da dor descontrolada. Estudo identificou que a dor é uma queixa comum de mais de $60 \%$ dos pacientes hospitalizados, não necessariamente em pós-operatório, mas com diagnósticos que incluem insuficiência respiratória aguda, exacerbação aguda de doença pulmonar, doença cardíaca congestiva e falência múltipla de órgãos ${ }^{(19)}$. Procedimentos como mudança de decúbito, curativos em feridas, punções em acessos centrais e periféricos, aspiração traqueal, entre outros, aos quais os pacientes podem ser submetidos durante a internação, também acabam por causar algum grau de dor ${ }^{(20)}$.

A dor deve ser investigada de forma multidimensional, principalmente, os fatores a ela relacionados. A demonstração de dor pode ser subjetiva e cabe aos profissionais, em especial, enfermeiros que estão diretamente envolvidos com o cuidado, considerarem os diferentes aspectos que fazem parte da experiência dolorosa. É de suma importância que os fatores relacionados à dor, bem como características que a evidenciam, sejam identificados o mais precocemente, para que intervenções pontuais sejam realizadas para proporcionar o alívio. Revisão integrativa que realizou definição conceitual e operacional do DE "Dor aguda" trouxe elementos que auxiliam o Enfermeiro a identificar o paciente com dor, sendo alguns deles: o autorrelato da presença de dor; o autorrelato de características da dor; comportamento de distração; comportamento expressivo, como choro, agitação e vigilância; comportamento protetor; desesperança; diaforese; dilatação pupilar; evidência de dor usando instrumento padronizado, para pessoas incapazes de se comunicar verbalmente; expressão facial de dor; mudanças no apetite; posição para aliviar dor; entre outros ${ }^{(20)}$. Destacase a realização de uma análise que considere também aspectos relacionados à idade, fragilidade emocional e identidade cultural dos pacientes, pois a dor pode estar disfarçada pelas particularidades de cada $\operatorname{ser}^{(21)}$.

Ao avaliar a intensidade da dor, apresentada pelos pacientes deste estudo, verificou-se que a maioria $(52,9 \%)$ queixou-se de dor moderada. Outros autores constataram que a queixa de dor moderada foi mais frequente, em pacientes do sexo masculino, em pós-operatório imediato (18\%), seguido de queixas de dor severa de máxima intensidade pela escala de avaliação da dor $(14 \%)^{(22)}$.

Detectou-se uma diferença na proporção de homens e mulheres de acordo com o nível de dor $(p=0,004)$. A proporção de pacientes que se queixaram dor leve e moderada foi maior entre os homens e somente mulheres se queixaram de dor severa. Outros autores observaram que a maioria dos pacientes com queixa de dor aguda era do sexo feminino $(65,5 \%)$ e houve maior frequência $(51,7 \%)$ da queixa de dor severa, nos participantes do estudo, independente do $\operatorname{sexo}^{(23)}$.

Ademais, estudo demonstrou evidências acerca da percepção da dor entre os dois sexos, mas as diferenças exatas e a sua relevância não são totalmente estabelecidas ${ }^{14}$. Um estudo sugere que mulheres apresentam maior frequência de episódios dolorosos, quando comparadas aos homens, pois elas estão mais susceptíveis a eventos patológicos que causam dor ${ }^{(15)}$. Entretanto é importante compreender que a dor pode acometer indivíduos de qualquer idade, sexo e condições socioeconômicas, cabendo à equipe de enfermagem realizar uma avaliação abrangente da dor e utilizar o raciocínio clínico e o PE para intervir e proporcionar um desfecho positivo aos pacientes.

Verificou-se uma correlação entre o tempo de internação e o status do DE "Dor aguda" no momento da alta. Quanto maior o tempo de internação maior o número de pacientes que tiveram o DE resolvido $(r=0,384 ; p=0,025)$. $O$ tempo médio de internação, observado neste estudo (3,97 dias), mostrou-se menor que o tempo encontrado por outros autores ( 8,5 dias), quando se trata de unidades de internação em clínica médica ${ }^{(13)}$. Porém é importante ressaltar que a unidade de internação, no cenário deste estudo, considera também que uma clínica cirúrgica recebe pacientes em pré e pósoperatório, especialidade que requer menor 
tempo de internação e alta precoce quando comparado aos pacientes da clínica médica.

Os pacientes que permaneceram por mais tempo, nas clínicas médica e cirúrgica, tiveram alta sem queixa de dor, quando comparados aos pacientes internados por um período mais curto. 0 relato de dor é uma característica marcante, em pacientes de pós-operatório, porém é comum que o paciente receba alta hospitalar, ainda, com queixa de dor, pois sua recuperação se dará, em maior parte, no ambiente domiciliar ${ }^{(17)}$. Quando se trata do paciente admitido em clínica médica, a alta acontece, mediante a resolução da maior parte das queixas, a incluir a dor, o que sustenta $o$ achado deste estudo.

Um estudo que avaliou a dor aguda, em pacientes admitidos na emergência, verificou que, no momento da primeira avaliação, $62,5 \%$ apresentavam dor intensa e $37,5 \%$ relataram dor moderada. Porém, em uma segunda avaliação, houve predomínio da queixa de dor moderada $(54,1 \%)$ e aumento significativo no número de pacientes com dor leve, demonstrando que intervenções adequadas, durante o tempo de internação, têm grande eficácia no controle da dor $^{(15)}$.

A comunicação verbal é considerada padrão-ouro, para uma avaliação efetiva da dor, sendo a incapacidade de se comunicar um obstáculo para a identificação e manejo adequado da dor nos pacientes hospitalizados ${ }^{(20)}$. Uma revisão integrativa demonstrou que, em alguns países, o relato verbal de dor pelo próprio paciente ou acompanhante tem sido utilizado, para a avaliação da dor, sem um instrumento apropriado. Contudo, em outros lugares, inclusive em alguns cenários brasileiros, percebe-se a avaliação da dor utilizando instrumento padronizado para este fim, a exemplo da escala visual numérica e analógica ${ }^{(20)}$.

No Reino Unido, por exemplo, apesar de não existirem programas específicos nos hospitais, para otimizar o manejo da dor, os profissionais realizam várias atividades de conscientização e educação sobre a dor, fazendo com que os pacientes reportem-na com mais frequência ${ }^{(19)}$. Nos EUA, já foram introduzidos programas de gerenciamento da dor para todos os pacientes hospitalizados. O mesmo ocorre no Canadá, onde são exigidos padrões mínimos semelhantes de avaliação e manejo da dor ${ }^{(19)}$. Uma revisão integrativa apontou lacunas de conhecimento sobre a avaliação e manejo da dor pela enfermagem em cuidados críticos pelo mundo ${ }^{(20)}$, o que sugere que a avaliação da dor pode ser ainda mais desvalorizada, em ambientes de cuidados mínimos e intermediários, em que os recursos humanos e materiais são mais escassos, como, por exemplo, no cenário deste estudo.

Destaca-se que o uso do SIPETI auxiliou na identificação do DE "dor aguda" em 34 pacientes, bem como avaliação diária do paciente com queixa de dor à beira do leito de maneira integral. Foi possível avaliar não apenas a intensidade da dor apresentada pelos pacientes, mas também outros aspectos envolvidos nesta resposta humana, como a localização, tipo e fatores relacionados. Em uma revisão integrativa, foi identificado que enfermeiros têm demonstrado atitude positiva, em relação ao uso de softwares, que auxiliam na aplicação do PE na prática clínica ${ }^{(24)}$. A partir da utilização de sistemas informatizados, verificou-se que o tempo despendido no atendimento foi menor, além da melhora no acolhimento do paciente e criação de planos de cuidado individuais ${ }^{(24)}$.

\section{CONSIDERAÇÕES FINAIS}

O presente estudo observou, no período de dois meses consecutivos, que a dor esteve presente em $34 \%$ dos pacientes. A maioria dos participantes foi do sexo masculino $(19-55,9 \%)$ e com idade inferior a 60 anos. Como causa da internação, os sistemas orgânicos gastrintestinal e musculoesquelético (9-26,5\%) foram os mais prevalentes e a maioria dos pacientes evoluiu para alta hospitalar. Do total de pacientes com o DE "Dor aguda", 21 (61,8\%) mantiveram o problema no momento da última avaliação. Apenas metade dos participantes teve acesso a medidas farmacológicas para o alívio da dor. $\mathrm{Na}$ maioria dos pacientes (19-55,9\%), a dor esteve relacionada a agentes físicos. Quanto maior o tempo de internação, melhores os prognósticos em relação à dor.

O paciente hospitalizado passa por uma fase delicada que pode ser acentuada pela vivência de dor, tendo a equipe de enfermagem papel importante em seu controle. Os profissionais devem utilizar escalas e instrumentos que permitam a avaliação abrangente da dor, considerando a individualidade de cada paciente e valorizando suas queixas. A capacitação do enfermeiro acerca da avaliação da dor deve se iniciar, durante a formação, a fim de aumentar o conhecimento sobre seus fatores relacionados e proporcionar o uso adequado de instrumentos que permitem 
quantificá-la. O PE não deve ser dissociado da prática clínica da enfermagem, por meio do qual é possível criar um plano de cuidados específico e individual, a fim de programar ações resolutivas no controle da dor.

Este estudo teve como limitação o tamanho da amostra que não permite a extrapolação de achados para populações maiores ou com perfil diferenciado. Espera-se que os achados deste trabalho possam contribuir, para a maior discussão sobre o tema, assim como melhor preparo da equipe de enfermagem, para realizar a avaliação e manejo adequado da dor, em quaisquer cenários de assistência, considerando a individualidade dos pacientes que buscam pelo serviço de saúde com queixa de dor ou que a apresentam em determinados momentos da hospitalização.

Ademais, os dados encontrados neste estudo possibilitaram caracterizar o DE "Dor aguda", nas unidades de clínica médico-cirúrgica e, assim, corroboram com os achados de outros autores no que tange à importância da avaliação da dor e intervenções adequadas em seu manejo.

NOTA: O Projeto foi financiando pela FAPEMIG: PROJETO N ${ }^{\circ} 2290-2016$.

\section{REFERÊNCIAS}

1- Merskey H, Bogduk N. Classification of chronic pain: descriptions of chronic pain syndromes and definitons of pain terms. 2nd ed. Seattle: IASP Press; 1994.

2- Sousa FAEF, Silva TCR, Siqueira HBOM, Saltareli S, Gomez RRF, Hortense P. A dor desde a perspectiva do ciclo de vida: Avaliação e medição através de métodos psicofísicos de estimação de categoria e magnitude. Rev Latino-Am Enferm. 2016;24:1-9. DOI: 10.1590/1518-8345t.0714.2769 3- Queiróz DT, Carvalho MA, Carvalho GD, Santos SR, Moreira AS, Silveira MF. Dor - 5o sinal vital: Conhecimento de enfermeiros. Rev Enferm UFPE 2015;9(4):7186-92. DOI: 10.5205/reuol.727562744-1-SM.0904201501

4- Silva PO, Portella VC. Nursing interventions in pain. Rev Dor 2014;15(2): 145-8. DOI: 10.5935/1806-0013.20140027

5- Herdman TH, Kamitsuru S. Diagnósticos de enfermagem da Nanda: Definições e classificação 2018-2020. Porto Alegre: Artmed; 2018.

6- Tannure MC, Lima APS, Oliveira CR, Lima SV, Chianca TCM. Processo de Enfermagem: comparação do registro manual versus eletrônico. J Health Inform. 2015 [citado em 8 jun
2019]; 7(3):69-74. Disponível em: http://www.jhisbis.saude.ws/ojs-jhi/index.php/ihi-

sbis/article/view/337.

7- Lobiondo-Wood G, Haber J. Pesquisa em enfermagem: Métodos, avaliação crítica e utilização. Rio de Janeiro: Guanabara-Koogan; 2001.

8- Coluci MZO, Alexandre NMC, Milani D. Construção de instrumentos de medida na área da saúde. Ciênc Saúde Coletiva 2015;20(3):92536. DOI: $10.1590 / 1413-81232015203.04332013$

9- Huskisson EC. Measurement of pain. Lancet 1974;2(7889):1127-31. DOI: 10.1016/s01406736(74)90884-8

10- Organização Mundial da Saúde (OMS). Alívio da dor e tratamento paliativo no câncer. Relatório de um comitê de especialistas. Genebra: OMS; 1990.

11- Vasconcelos AC, Castro CGA, Silva DF, Sousa VJ. Frequência de diagnósticos de enfermagem em uma clínica cirúrgica. Rev Rene 2015;16(6):826-32. DOI: $10.15253 / 2175-$ 6783.2015000600008

12- Novaes ES, Torres MM, Oliva APV. Diagnósticos de enfermagem em clínica cirúrgica. Acta Paul Enferm. 2015;28(1):26-31. DOI: 10.1590/1982-0194201500006

13- Lopes EB, Pupulim JSL, Oliva APV. Perfil dos diagnósticos de enfermagem de pacientes internados em unidade de clínica médica. Cienc, Cuidado Saúde 2016;15(2):358-65. DOI: 10.4025/cienccuidsaude.v15i2.29356

14- Figueiredo W, Alvez T. Uso dos antiinflamatórios não esteroides no controle da dor aguda: Revisão sistemática. Rev Neurociênc. 2015;23(3):463-7.

DOI: 10.4181/RNC.2015.23.03.1070.05p

15- Bertoncello KCG, Xavier LB, Nascimento ERP, Amante LN. Dor aguda na emergência: Avaliação e controle com o instrumento de MacCaffery e Beebe.J Health Sci. 2016;18(4):251-6. DOI: 110.17921/2447-8938.2016v18n4p251-6

16- Matos A, Cardoso R, Coisinha S, Silveira S, Lotra $V$, Fonseca $C$. Medidas não farmacológicas na pessoa com dor: resultados sensíveis da intervenção dos enfermeiros. Revisão sistemática da literatura. RIASE 2017;3(3):1198-216. DOI: 10.24902/r.riase.2017.3(3).1198

17- Meier AC, Siqueira FD, Pretto CR, Collet CF, Gomes JS, Dezordi CCM, et al. Análise da intensidade, aspectos sensoriais e afetivos da dor de pacientes em pós-operatório imediato. Rev Gaúcha Enferm. 2017;38(2):1-8. DOI: $\underline{10.1590 / 1983-1447.2017 .02 .62010}$ 
18- Liu D, Ma J, Zhang Z, Ailan A, Chen X, Feng C, et al. Management of postoperative pain in medical institutions in Shandong Province in China. Medicine 2016;95(6): e2690. DOI: 10.1097/MD.0000000000002690

19- Gregory J, McGowan L. An examination of the prevalence of acute pain for hospitalised adult patients: a systematic review. J Clin Nurs. 2016;25(5/6):583-98. DOI: 10.1111/jocn.13094

20- Correia MDL, Duran ECM. Conceptual and operational definitions of the components of the nursing diagnosis Acute Pain (00132). Rev LatinoAm Enfermagem. 2017;25:e2973. DOI: $110.1590 / 1518-8345.2330 .2973$

21- Martin AR, Soares JR, Vieira VCL, Marcon SS, Barreto MS. A dor aguda na perspectiva de pacientes vítimas de trauma leve atendidos em unidade emergencial. Rev Gaúcha Enferm. 2015;36(2):14-20. DOI: $\quad$ 10.1590/19831447.2015 .02 .48728

22- Salgado PO, Lima TM, Souza CC, Toledo LV. Características definidoras do diagnóstico de enfermagem "dor aguda" em pacientes no pósoperatório imediato. Enferm Rev. 2017 [citado em 15 jan 2019]; 20(3):66-80. Disponível em: http://periodicos.pucminas.br/index.php/enferm agemrevista/article/ view/17232

23- Sousa-Muñoz RL, Rocha GES, Garcia BB, Maia $A D$. Prevalência de dor e adequação terapêutica analgésica em pacientes internados em um hospital universitário. Medicina 2015;48(6):53948. DOI: $10.11606 /$ issn.2176-7262.v48i6p539-548 24- Domingos CS, Boscarol GT, Brinati LM, Dias AC, Souza CC, Salgado PO. A aplicação do processo de enfermagem informatizado: revisão integrativa. Enferm Glob. 2017;16(48):603-52. DOI: $\underline{10.6018 / \text { eglobal.16.4.278061 }}$

Nota: Este estudo faz parte do trabalho de conclusão de curso intitulado "Avaliação do diagnóstico de enfermagem "dor aguda" em pacientes internados em uma clínica médico-cirúrgica", para obtenção do título de Bacharel em Enfermagem pela Universidade Federal de Viçosa (UFV), Campus de Viçosa. Pesquisa financiada pela Fundação de Amparo à Pesquisa de Minas Gerais-FAPEMIG № 2290-2016.

Recebido em: 13/03/2019

Aprovado em: 25/09/2019

Endereço de correspondência:

Gabriela Tavares Boscarol

Rua Virgílio Val, 270 - Centro

CEP: 36570-023 - Viçosa/MG - Brasil

E- mail: gabiboscarol@gmail.com 